\title{
Whole Body Interaction in Abstract Domains
}

\author{
Simon Holland, Katie Wilkie, Anders Bouwer, Mat Dalgleish and Paul \\ Mulholland \\ The Music Computing Lab \\ Department of Computing \\ The Open University \\ Milton Keynes \\ UK \\ \{s.holland, k.1.wilkie, \\ p.mulholland\}@open.ac.uk \\ Intelligent Systems Lab \\ Informatics Institute, \\ Faculty of Science \\ University of Amsterdam \\ The Netherlands \\ a.j.bouwer@uva.n \\ Department of Music \\ (SPAL) \\ University of \\ Wolverhampton \\ Gorway Road \\ Walsall \\ UK \\ m.dalgleish2@wlv.ac.uk
}

\section{INTRODUCTION}

There is little dispute that Whole Body Interaction is a good fit of interaction style for some categories of application domain, such as the motion capture of gestures for computer games and virtual physical sports. This reflects the observation that in such applications the mapping between user gesture and the desired effect is, broadly speaking, the identity function. For more abstract application areas such as mathematics, programming and musical harmony, finding appropriate mappings between gesture and effect is less straightforward. The creation of appropriate whole body interaction designs for such abstract application areas remains challenging. However, this is not to argue that whole body interaction is unsuited to abstract domains. Indeed, there is evidence, outlined below, that whole body interaction offers excellent affordances for some highly abstract applications areas.

In this chapter we consider an argument to this end, derived from conceptual metaphor theory and sensory motor contingency theory, concerning the suitability of whole body interaction to abstract application domains in general. In particular, we analyse a case study of a whole body interaction system for a highly abstract application area, advanced tonal harmony. This domain is generally considered challenging, irrespective of how it is approached, but whole body interaction appears to offer particularly interesting affordances for action and insight in this domain, when appropriate conceptual metaphors and conceptual blends (see below) are harnessed in the design using whole body interaction. We analyse issues emerging from this case study and consider implications of these issues for whole body interaction design when dealing with highly abstract domains in general. 


\section{Conceptual Metaphor and Embodied Experience}

Conceptual Metaphor theory is an important strand in current research on Embodied Cognition (Klemmer et al., 2006). Conceptual Metaphor Theory (Lakoff and Johnson, 2003; Lakoff and Nunez, 2000; Johnson, 2005; Rohrer, 2007) posits that human capabilities for dealing with abstract concepts are always grounded in a small core of universal sensory-motor abilities, together with their associated lowlevel inferencing capabilities. More specifically, the theory hypothesizes that all cognitive capacities for dealing with abstraction are constructed by a process of association, mapping, composition and extension of maps, applied not just to entities, but also to roles, relationships and specialised inference capabilities, and grounded universally in low-level sensory motor resources for dealing with the body, objects, space and force (Lakoff and Nunez, 2000).

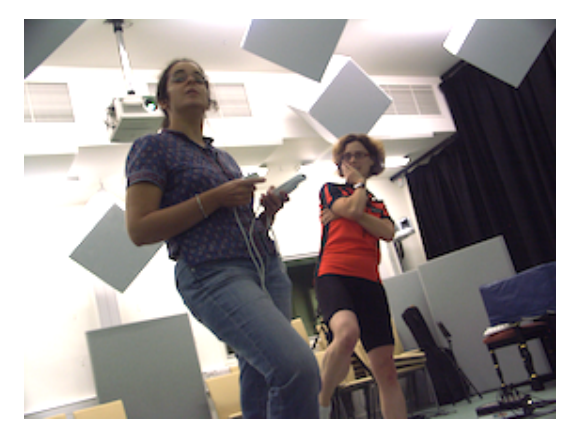

Figure 1. A case study in Whole Body Interaction, described in this chapter. Different players play contrasting, but interacting, roles.

Suggestive evidence for this theory comes from Linguistics and other areas of Cognitive Science including Psychology, Neuroscience and Human Computer Interaction (Lakoff and Nunez, 2000). Conceptual Metaphor Theory has been applied variously in Mathematics (ibid.), Political Analysis (Lakoff, 2002), Human Computer Interaction (Hurtienne and Blessing, 2009) Music Theory (Zbikowski, 1998; Saslaw, 1996; Larson, 1997; Johnson and Larson, 2003) and Music Interaction Design (Wilkie and Holland, 2009) and Interactive Sound Generation (Antle, Droumeva, and Corness 2008; Antle, Corness, and Droumeva 2009). For an application to issues of balance in the abstract domain of social justice, see Antle et al. in this volume. Mathematics teachers have judged the theory insightful and useful in teaching abstract mathematical concepts (Gold, 2001), and the theory has given rise to forms of analysis in HCI that have demonstrated that image schemas can help with design decisions for user interfaces (Hurtienne and Blessing, 2009; Hurtienne et al 2008). 
Broadly, there are three fundamental resources described in the theory: innate cognitive capacities, sensory motor schema, and conceptual metaphors. Innate cognitive capacities, such as subitizing ${ }^{1}$, grouping, pairing, and exhaustion detection, are shared with babies and some animals (Lakoff and Nunez, 2000). Sensory motor schema, also known as image schema, such as CONTAINMENT, CONTACT, CENTRE-PERIPHERY, and SOURCE-PATH-GOAL ${ }^{2}$ are associated with various kinds of low-level, special-purpose inference (ibid). Conceptual metaphors enable the two already mentioned classes of fundamental cognitive resource to be applied to domains of arbitrary complexity by mapping innate cognitive capacities and image schemas onto more abstract domains. Examples of employment of conceptual metaphors include reasoning about abstract classes (based, in part, on concrete lowlevel reasoning about containers) and reasoning about arithmetic (based, in part, on innate reasoning about collections of objects).

Conceptual metaphors can be applied in two different ways: as grounding metaphors, and in conceptual blends. Grounding metaphors enable the simplest abstractions to be grounded directly in physical experience - for example arithmetic subtraction as grounded by taking objects away from a collection, or mathematical sets as grounded in experience of collections of objects. Conceptual blending works by allowing two or more conceptual metaphors to be used to create new expanded or composite metaphors by processes of composition, completion and elaboration (Fauconnier, Gilles and Turner, 2002). These processes allow conceptual metaphors such as NUMBERS ARE COLLECTIONS OF OBJECTS and NUMBERS ARE LENGTHS to be blended in intricate ways, as analysed in detail in Lakoff and Nunez (2000), to produce more highly developed abstract concepts such as real numbers, complex numbers and transfinite number systems.

While image schema and innate capabilities such as subitizing are considered universal, conceptual metaphors can, and do, develop differently between different individuals and cultures. The formation of conceptual metaphors can be affected by a combination of innate, developmental, individual and cultural factors.

At present, empirical evidence for the validity of conceptual metaphor theory is growing, if not conclusive. More empirical work is needed to establish the limits within which the various elements of the theory validly apply. However, there is good evidence from Mathematics Teaching, Psychology, Human Computer Interaction and Linguistics that conceptual metaphor theory is at the very least pragmatically useful, for example to teachers and designers. In this chapter, we apply

\footnotetext{
${ }^{1}$ Subitizing is the ability to make judgements, without counting, about numbers of items up to about 3 or 4 . It has been empirically demonstrated that this ability is present in babies and some animals. It appears that different brain areas are used for subitizing vs. counting. Subitizing is claimed to be one foundation, via conceptual metaphor and blending, for the concepts of number and arithmetic (Lakoff and Nunez, 2000).

2 A common convention is to capitalize the names of schema and conceptual metaphors.
} 
the theory to illuminate a case study in order to explore general implications for whole body interfaces for abstract domains.

\section{A complex abstract domain: Tonal Harmony}

Tonal Harmony concerns the organisation of multiple simultaneous pitch sources, for example, two or more singers, or instrumentalists, playing independent but co-ordinated melodic lines. Tonal Harmony is generally considered the most technical part of music theory, requiring the mastery of demanding practical skills. Explicit understanding of tonal harmony involves understanding a wide range of abstract entities, relationships and terminology. These concepts span interlinked semi-hierarchical conceptual levels (for example, pitch, interval, scale, chord quality, voicing, mode, key and modulation). Each of these levels is associated with its own technical vocabulary. As well as being technically and conceptually demanding, tonal harmony is associated with practical obstacles to mastery. Generally, it is difficult for individuals without instrumental skills to get real-time experience of controlling multiple voices simultaneously in a flexible but precise manner - since without instruments or collaborators, we can only directly control a single voice. This is not the only route to mastery, but it is an important one.

To see how this relates to whole body interaction and conceptual metaphor, it will help to briefly consider two related theoretical perspectives, namely Sensory Motor Contingency Theory and Dalcroze Eurhythmics.

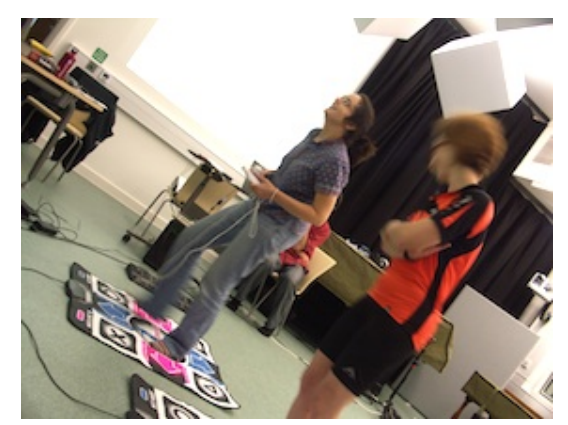

Figure 2. Dance mats and Wii controllers as used in the case study help to engage physical, spatial and navigational intuitions with the spatial conceptual metaphors reflected in the interaction design (described below). 


\section{Sensory motor contingency theory}

Sensory motor contingency theory (O'Regan and Noe, 2001) suggests that in order to learn to organize and respond appropriately to sensory input in some new domain or context, it is vital that one's motor actions have the power to perceptibly affect relationships in that domain. In diverse situations where this specific kind of feedback and experience is absent, competency has been observed to fail to develop. This principle has been demonstrated in numerous different contexts and time scales. Notable examples include the development of the visual perception systems of neonate kittens (Held and Hein, 1958) systematic adaptation to sensory substitution in blind adults over months (Bach-y-Rita, 2005); and opportunistic learned sensory substitution in sighted adults over minutes when carrying out simple tracking tasks (Bird et al., 2008).

Sensory motor contingency, assuming its applicability to the present case study, suggests that if we wish learners to develop their skills in engaging with, recognizing, identifying, memorizing, analyzing, reproducing, adapting and composing harmonic sequences, then their motor actions must have the capacity to actively manipulate those harmonic sequences to adequately fine levels of detail. However, in the domain under consideration, most novices cannot play musical instruments, and even if they can sing, singing only allows control of a single melodic line - not multiple voices. This argument can be taken too far. Trained singers, and players of single voiced instruments may be able to gain active experience of harmony by effectively multiplexing a single voice into multiple lines, or by mentally manipulating virtual lines. However, for those who cannot play a musical instrument and who are not active singers, conventional approaches to teaching harmony using books and passive listening may leave them at a distinct disadvantage, by a lack of experience of active control of the phenomenon of interest.

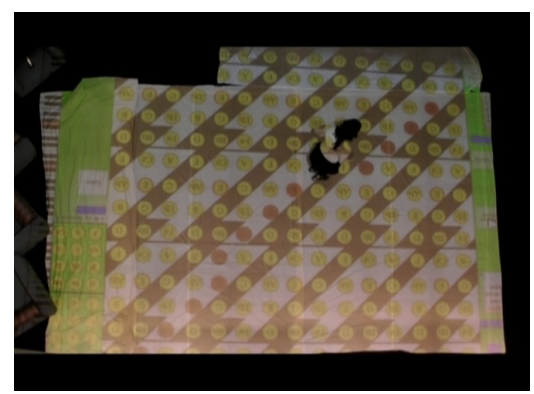

Figure 3. An earlier whole body version of Harmony Space designed to use floor projection and camera tracking. 


\section{Lessons from Dalcroze Eurhythmics}

A related theoretical perspective, Dalcroze Eurhythmics, applies to musical rhythm rather than harmony, but shares key issues with tonal harmony. The music educator Emil Dalcroze (1865-1950) noticed that his students seemed unable to deal with technical and written aspects of rhythm if they lacked experience of enacting and feeling the relevant rhythms with their bodies. Simply hearing examples did not appear to be sufficient. Dalcroze proposed that students had to become competent in enacting representative rhythms bodily. Once this had been achieved, problems in dealing with technical and abstract aspects of rhythm were readily solved. In some cases, the requirement for bodily enacting a rhythm can be relatively undemanding, for example if the rhythm can be tapped with a single finger (a monophonic rhythm). However, some rhythms involve more than one independent stream of sounds, and are considerably harder to recognize, memorize, analyze and reproduce than monophonic rhythms (Holland et al., 2010).

In order to encourage competency in enacting rhythms, particularly ones involving multiple streams, Dalcroze invented a system of rhythmic 'gymnastics' or 'eurhythmics'. Amongst other things, this involved asking students to walk at a regular pace, while moving their arms in synchrony at, for example, twice or three times the rate.

We propose that these insights from Dalcroze Eurhythmics can be applied fairly straightforwardly in principle to tonal harmony. Students typically seem unable to deal securely with technical and written aspects of music connected with harmony if they lack experience of enacting, composing and modifying sequences of simultaneous pitches in real time. Simply hearing examples does not appear to be sufficient. We propose that to develop skills in harmony students need experience of enacting and manipulating representative harmonic sequences with their own bodies.

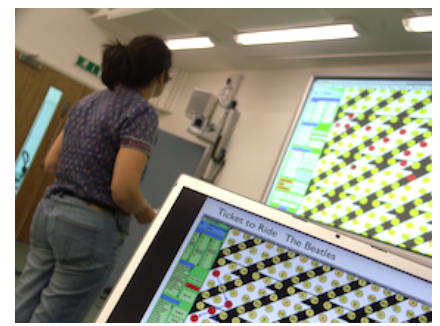

Figure 4. A player navigating a harmonic space 


\section{Harmony Space - a system for exploring tonal harmony}

Harmony Space (Holland, 1989; 1994) is an interactive digital music representation system designed to give beginners and experts insight into a wide range of musical tasks ranging from performance and analysis to composition. When combined with whole-body interaction to create the Song Walker version (Holland et al., 2009), Harmony Space allows users to enact complex harmonic phenomena physically via mappings between a) bodily movement and b) conceptual metaphors and blends for musical abstractions. This approach affords rapid, concrete means of reasoning about and manipulating abstract musical entities and relationships by exploiting intuitions associated with bodily movement and navigation.

\section{System details}

A previous design study (Holland et al., 2009) explored a version of whole body Harmony Space designed to work with camera tracking and floor projection (Figure 3) However, we sought a simpler, more portable version that could nonetheless afford a similar degree of physical engagement. The Song Walker version employs four dance mats, four wireless controllers (wii remotes and nunchucks), various foot pedals, a large projection screen and a synthesizer. These are coordinated by a Harmony Space Server receiving data from controllers via HSp (Harmony Space protocol, a layer on top of OSC (Open Sound Control).

When operated by a solo player, one dance mat is used to navigate a proxy for the player (represented by crosshairs in the wall projection) around in the projected space. Squeezing the trigger on the wii remote visibly and audibly plays the chord associated with the current location and situation. When operated by multiple players, additional dance mats may be used simultaneously in a variety of collaborative ways, for example, to navigate key changes or to harmonically invert or otherwise alter the chords.

\section{Conceptual metaphors and blends in Harmony Space}

Harmony Space exploits a set of conceptual metaphors that link concepts in tonal harmony to spatial concepts. Many of these are elaborations of the postulated basic conceptual metaphor of pitch: pitch intervals are displacements in three dimensions. This conceptual metaphor stems from Longuet Higgins' (1962) and Balzano's theories (1980) of harmonic perception, which retrospectively may be seen as positing a three-dimensional image schema for tonal harmony. As previously noted, conceptual metaphors typically emerge from a combination of innate, devel- 
opmental, individual and cultural factors (though image schemas themselves are generally universal). Rich elaborations of the basic conceptual metaphor of pitch were afforded by early versions of Harmony Space in a combined technical, and cultural process in three stages, as follows.

a) Harmony Space introduces and embodies a set of metaphorical blends in which the basic conceptual metaphor of pitch interacts with itself in a series of layers. These represent interacting abstract layers of harmony; pitch alphabets, scales, keys, chord functions, tonal centres, roots, and bass lines, unified by the basic conceptual metaphor (Holland, 1989).

b) The interactive digital nature of the tool facilitates the visual plotting of diverse music from the repertoire in spatial terms. This process affords reflection by interested users and music analysts on the emerging patterns and their musical meaning. Some patterns correspond to well known analytic concepts. Others have given rise to novel analytic and compositional insights.

c) From the above technical and cultural process, new ways of discussing the technicalities of harmonic phenomena have emerged using a spatial, movementbased vocabulary as an alternative and complement to symbolic technical terms. Some important candidate conceptual metaphors and blends employed in Harmony Space, both in the desktop and whole body versions, are outlined below .

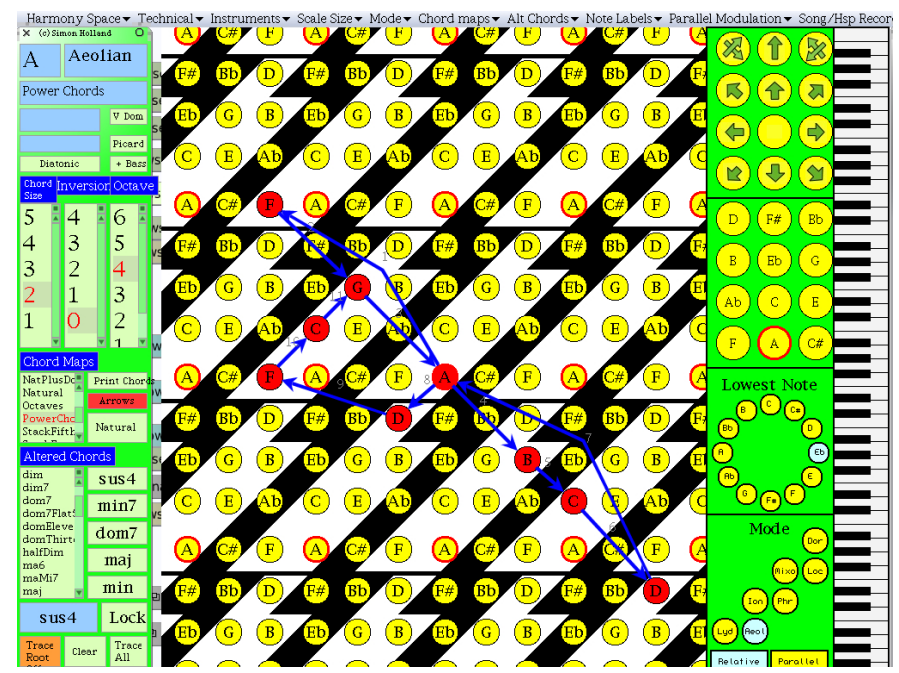

Figure 5 A root trajectory showing David Bowie's Suffragette City, used in an analytical task. The white shaded 'preferred territory' is physically moved under the red nodes and blue arrows that trace out the movement of the song, until the white territory fully contains the red nodes.

The following is an early attempt to identify and frame candidate conceptual metaphors for this domain. Some may prove to be overly specific, framed at the wrong level, or not truly universal. The current statements do not clearly distinguish candidate conceptual metaphors and surrounding explanation. For these reasons, can- 
didates are not capitalized, as confirmed conceptual metaphors would be by convention. Still, this initial draft provides a basis for search, criticism, refutation and refinement. We believe this is a step towards illuminating the rich conceptual metaphorical structure of harmony.

\section{Pitch}

- Different musical intervals (octave, semitones, perfect fifths, major thirds, minor thirds) correspond to steps in different directions in space.

- In particular, semitones, fifths, and octaves are at right angles to each other in the plane, and octaves lie vertically (i.e., on the z-axis).

- The two main axes in the plane (semitones and perfect fifths) each have their own local vertical sense in the plane (this is an example of a conceptual blend).

- When moving in a straight line in any of the eight main directions in the plane, locations are repeated (depending on direction, this happens after 3, 4 or 12 steps).

\section{Scales, keys and modes}

- Common scales can be formed from the notes occurring in contiguous strips seven steps long in the fifths direction. Due to the repeating nature of the plane, these strips group into irregularly shaped two dimensional areas (as illustrated in figure 5).

- Key areas are scales spatially situated to represent preferred territory for journeys and chord elements (see the white area in figure 5).

- Diatonic materials are materials that restrict their elements and movement to preferred territory.

- Modulation is the co-ordinated movement of a preferred territory.

- A modal centre is a privileged location within preferred territory, typically where journeys start, end or rest.

- Mode changes occur when the privileged location moves within its enclosing territory.

\section{Chords}

- The most common chords (stacked thirds chords, as found in musical styles varying from classical and folk music to blues and jazz) are collections of two or more objects maximally compactly by local gravity to fit within a preferred territory.

- Triads are three element chords. Seventh chords are four element chords. Ninth chords are five element chords.

- Chord qualities are oriented geometrical shapes. Preservation of chord quality requires retention of shape and orientation. Altering the pitch of a fixed quality is change of location of the shape without rotation.

- The most common chord qualities, major and minor, correspond to the most frequent three-element chord shapes formed by compaction within the geometry of the most common scales. 


\section{Key Centres}

- The geometry of diatonic materials causes major and minor chord qualities to occur in groups within preferred territories. The spatial centres of these groups are the major and minor key centres (see Brower (2000) for other perspectives).

\section{Harmonic Functions}

- Different harmonic functions are associated with different locations within a territory.

- Due to the irregular shape of key areas, different chord qualities fit within the preferred territory at different locations. Thus different harmonic functions are associated with different chord qualities.

Inversion and voicing

- Inversion and voicing are movements of chord elements on the z-axis, displacing elements by an octave.

\section{Harmonic movement}

- Harmonic movement is spatial trajectory.

- Chord sequences, bass lines, and modulations are journeys.

- Composition is navigation, which may involve targets, directions, inertia, oscillatory movement and preferred territories.

- Due to the recurrence of locations in the plane, identical destinations may be reachable by alternative routes. Different routes correspond to different harmonic analyses.

- Journeys may segment in one way when viewed geometrically, but in another way when compared with an external clock ("Harmony vs. Hypermeter").

- Tonal movement corresponds to trajectories along the diagonal from top right to bottom left or vice versa.

- Modal movement corresponds to trajectories along the diagonal from top left to bottom right or vice versa.

- Tonal and modal movement are at right angles to each other.

- Tonal and modal tensions can be adjusted by swerves in direction.

- Tonal and modal ambiguity arise where direction of movement and location of territory is ambiguous.

The above candidate conceptual metaphors are arcane when expressed in words. They are more easily absorbed physically, visually and aurally. Their physical, spatial nature and their emphasis on navigation makes them well suited for enacting through whole body interaction. Furthermore, the occurrence of related interacting spatial metaphors at different levels (pitch, key, inversion, modality) offers opportunities for collaborative navigation. 


\section{Whole Body Interaction vs. desktop}

Harmony Space has existed previously principally as diverse desktop prototypes (Holland et al., 2009). Beneath the different user interaction layer, the semantics of the Song Walker and desktop versions are essentially identical. However, the types of reasoning elicited from users by the two versions appear to contrast interestingly in some respects.

When a user controls a harmonic sequence in real-time on the desktop system using a mouse and alphanumeric keyboard, some operations must be carried out on the keyboard more or less simultaneously. This requires a certain amount of learning and expertise about the details of the keyboard control system, which can get in the way of direct engagement with the domain. Furthermore, spatial movements of various kinds in the various dimensions of the harmonic domain are much more simply mirrored using dance mats and wii controllers than by alphanumeric key sequences and mice.

When two or more players control a harmonic sequence in real time using the dance mat version, appropriate simple spatial movements can be collaboratively taught with minimal preamble, with directly perceived musical results. Thus, the affordances offered by the design that relate to conceptual metaphors and conceptual blends in the conceptual structure of the domain can be enacted and experienced more directly using the dance mat version than a desktop version.

\section{Asymmetrical collaboration}

As well as the potential advantages of whole body interaction for engaging with spatial phenomena, there are also potential advantages for supporting collaborative roles.

Typically, when musicians collaborate to produce harmony, each musician contributes one part (which in some cases may contribute more than one voice - and in other cases single voices may be split between musicians). From many points of view, especially for experts, this more or less symmetrical distribution of roles is highly appropriate. However, where players are novices, or where there is a desire to gain insights into the abstract structures of tonal harmony, one drawback of the conventional approach is that that this process leaves these abstractions intangible and invisible.

By contrast, when Harmony Space is used collaboratively, the roles do not have to be split voice-wise, but may be split asymmetrically into heterogeneous spatial navigation and selection tasks, corresponding to abstractions of interest. 


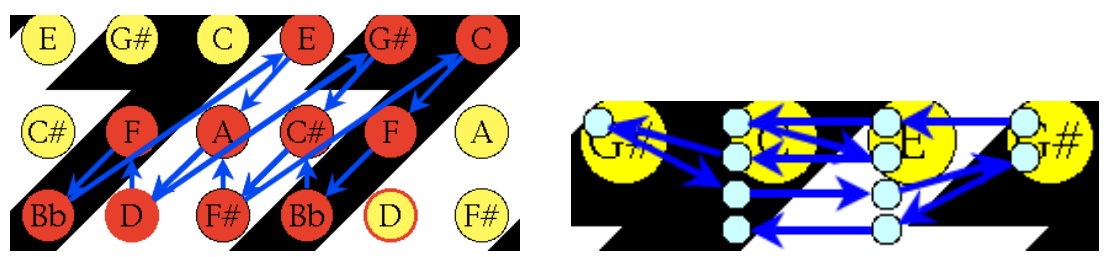

Figure 6. In the study, some players collaborated to play the chord sequence of John Coltrane's Giant Steps. The left hand figure shows the path navigated by one player to control the root path. The right hand figure shows the path navigated simultaneously by another player controlling the key changes. The trace of the performance corresponds to a detailed abstract harmonic analysis of the piece.

Contrasting simultaneous asymmetrical roles available include the navigation of: the root path; changes of key; inversions and voicing; chord size; chord maps; altered chords; and bass lines. For particular pieces of music, typically only two or three of these roles are required at a time. The combinatorial interplay of these factors yields the detail of harmonic sequences.

One key motivation for an asymmetrical approach is that experiencing these different roles allows players to enact aspects of the distinct conceptual metaphors whose blending together, we hypothesize, plays a key role in the understanding and mastery of tonal harmony. For example, movement in space can correspond under different metaphors variously to root movement and key movement. Deeper understanding comes from experiencing how these different kinds of movement can interact.

With multiple dance mats, players can readily see what other players are doing, and the rhythms to which they are working.

By rotating roles while performing, analyzing and composing, beginners can learn something about how different conceptual metaphors have been tacitly blended together in a variety of ingenious ways by composers over some 400 years.

\section{Empirical Observations}

Recent trials with eight pairs of users, some complete beginners, some musical experts, are being analyzed formally elsewhere. Here we limit ourselves to general observations. As regards the performance of pieces, all participants could perform one or more moderately complex songs after a few minutes training. Songs performed included:

- Ticket to Ride (The Beatles),

- Isn't She Lovely (Stevie Wonder),

- Pachelbel's Canon,

- Giant Steps (John Coltrane),

- Billie Jean (Michael Jackson). 
Participants asked to harmonically analyse a piece, such as Suffragette City by David Bowie, were able to do so. This task required identifying the mode of the piece by physically shifting territory by means of a dance mat until the trace of the harmonic journey fell entirely within the preferred territory (see figure 5).

All participants could compose and modify simple chord sequences in musically intelligent ways. In a simple-open ended collaborative navigational composition task, one pair of beginners spontaneously focused particularly on inversions and explored these carefully, with much discussion and multiple attempts. Another pair of users deployed altered chords in a similarly careful way to musically positive effect. The care with which most beginners collaboratively composed musically sensible chord paths was surprising. Several users commented on the degree of physical engagement they brought to the tasks.

To illustrate this, one initially skeptical user was able to learn to play the complete harmony of Pachelbel's canon after about ten minutes, At this point he said variously "I haven't got this musically in my head at all", "I don't have a sense of what's going on cognitively - how the visual representation is helping me remember it", and "visually overwhelming". However about 30 minutes later, having played several more songs, he commented "Love the kinaesthetic quality" and "Once you're used to it, you could dance songs" (in the sense that Song Walker allows one to generate the harmony for a song by dancing to it).

Comments on the degree of physical engagement might be unremarkable in the case of, for example, arcade games, but are unusual in the context of tasks that are generally taught in knowledge intensive ways using rule-based, symbolic, and quasi-mathematical approaches. Also, conventional approaches to learning these tasks generally take one or two orders of magnitude longer.

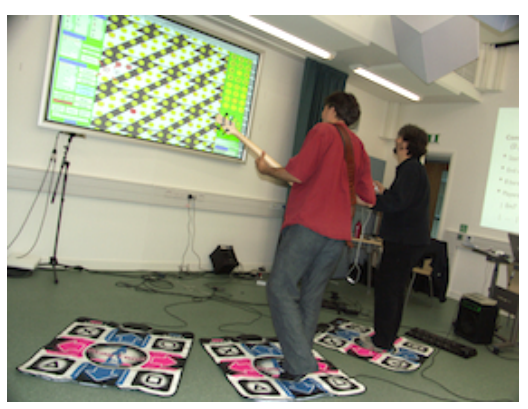

Figure 7. Two players improvise collaboratively using the whole body interface. The bass player plays his instrument normally and controls frequent key shifts with his feet with the dance mat. The second player navigates a chord sequence. Both players sometimes lead and sometimes follow. The game was played too fast for reflective thought and was considered highly engaging. 
The user trial was not designed to look for evidence of long-term adoption by participants of the conceptual metaphors enacted. However, there was anecdotal evidence that this happened. For example, days after the trial, one non-musically trained participant returned to discuss the harmonic structure of a song that he knew, but which had not featured in the trial. Without since having had any access to the interface, he correctly identified the path this song would trace, and went on to ask insightful questions about the musical implications of this path.

\section{Conclusions}

Taking into account the theoretical issues and the case study, what lessons can be drawn for whole body interaction in general? Let us marshal the key theoretical points to help us synthesize a concrete proposal. As already noted, conceptual metaphor theory posits that all human capabilities for dealing with abstract concepts are grounded in low-level sensory motor resources for dealing with the body, objects, space and force. More specifically, conceptual metaphors are developed by various developmental and cultural processes to allow abstract concerns to be manipulated using resources borrowed from universal image schemas. This allows abstractions to be grounded directly in physical experience. To go beyond this, and in order to be able to deal with abstraction of arbitrary complexity, we need conceptual blending to allow two or more conceptual metaphors to be melded by processes of composition, completion and elaboration. In all cases, an audit trail, from the abstractions back to physically grounded experience may be identified. This does not create a panacea for instantly simplifying any domain, but it does in principle relate any abstract topic to the physically-related, low-level skills to which humans are well suited.

It remains a non-trivial task to analyse conceptual metaphorical structures in any specific domain, though as noted, progress has been made in areas such as Mathematics, Human Computer Interaction, Music Theory and Harmony. How then, can this be applied to whole body interaction design in the general case? In effect, we are arguing for an elaboration of the approach to interaction design advocated by Dourish (2001). Like Dourish, we argue that collaborative, physically engaged interaction allows a wider range of human mental resources to be brought to bear on problems than sitting at a desk in isolation thinking abstract thoughts. In a difference of emphasis to Dourish, we see the direct exploitation of conceptual metaphors in interaction design as a particularly powerful way to bring these resources to bear on abstract problems domains. In particular we advocate the following key steps when designing for whole body interaction.

a) The crafting of user interaction metaphors specifically to reflect and exploit the physical grounding of conceptual metaphors and blends in the given domain. 
b) The exploitation of cues to prompt the user to foreground physically grounded mental resources in manipulating the conceptual metaphors and blends exploited by the interface.

The good news is that the previously rehearsed theoretical arguments suggest that by this process, it should be possible to usefully apply whole body interaction to any arbitrary abstract domain. Of course, limitations will apply. Zbikowski (1997) points out how conceptual metaphors can vary greatly in the density of imageschematic correspondences between source and target domains. The fewer the correspondences, the less useful a conceptual metaphor is likely to be. Strong conceptual metaphors can form the basis of more effective whole body interaction designs than weak ones. Zbikowski (1998) and Eitan and Timmers (2010) identify some rather weak conceptual metaphors in music, for example those concerning crocodiles and waterfalls, while Lakoff and Nunez (2000) identify some extraordinarily strong conceptual metaphors in mathematics, for example in their conceptual analysis of Euler's equation. The candidate conceptual metaphors in Harmony Space are highly dense (assuming they are validated) as many spatial metaphors are. However, we hypothesise that conceptual metaphors need not necessarily be explicitly spatial in nature to effectively support whole body interaction. What matters is that the density of image-schematic correspondences between source and target domains should be high, and the length of chains in conceptual blends not too long. Of course, the success of this approach will always remain dependent on the identification of conceptual metaphors in the domains of interest, as does the closely related approach that employs conceptual metaphors to analyse interaction designs, pioneered by Wilkie et al. (2010).

We offer the present case study as an existence proof of the value and reach of the explicit use of conceptual metaphors in whole body interaction design. We have demonstrated in at least one abstract domain that this approach affords the performance of complex technical tasks that otherwise require extensive abstract symbolic knowledge.

\section{References}

Antle, A. N., G. Corness, and M. Droumeva. 2009. "Human-Computer-Intuition? Exploring the Cognitive Basis For Intuition in Embodied Interaction.” International Journal of Arts and Technology 2(3): 235-254.

Antle, A. N., M. Droumeva, and G. Corness. 2008. "Playing With the Sound Maker: Do Embodied Metaphors Help Children Learn?" Proceedings of the 7th International Conference on Interaction Design and Children. New York: Association for Computing Machinery, pp. 178185.

Bach-y-Rita, P. Brain Mechanisms in Sensory Substitution. Academic Press, NY, 1972.Studies, 64 (1) (2005), 1-15. 
Balzano, G. J. (1980) The Group-theoretic Description of 12-fold and Microtonal Pitch systems. COMPUT MUSIC J, 4, 4 (Winter 1980), 66-84.

Bird, J., Holland, S., Marshall, P., Rogers, Y. and Clark, A. (2008) Feel the Force: Using Tactile Technologies to Investigate the Extended Mind. In Proceedings of Devices that Alter Perception Workshop (DAP 08).

Brower, C. 2000. "A Cognitive Theory of Musical Meaning." Journal of Music Theory 44(2):323-379.

Dourish, P. (2001) Where the Action Is: The Foundations of Embodied Interaction. MIT Press, Cambridge Mass. ISBN 978-0-262-54178-7.

Eitan, Z., and R. Timmers. 2010. "Beethoven's Last Piano Sonata and Those Who Follow Crocodiles: Cross- Domain Mappings of Auditory Pitch in a Musical Context." Cognition 114(3):405-422.

Fauconnier, Gilles and Turner, Mark. 2002. The Way We Think: Conceptual Blending and the Mind's Hidden Complexities. Basic Books.

Gold, B. (2001) Mathematical Association of America Online Book Review. http://www.maa.org/reviews/wheremath.html

Held, R. and Hein, A. V. (1958) Adaptation of disarranged hand-eye coordination contingent upon re-afferent stimulation. Perceptual and Motor Skills. Vol 8, 1958, 87-90.

Holland, S. (1989). "Artificial Intelligence, Education and Music.” PhD Thesis, Institute of Educational Technology, The Open University, Milton Keynes, England.

Holland, S. (1994) Learning about harmony with Harmony Space: an overview. In Smith, M. and Wiggins, G. eds. Music Education: An Artificial Intelligence Approach, 1994.

Holland, S., Marshall, P., Bird, J., Dalton, S., Morris, R., Pantidi, N., Rogers, Y. and Clark, A. (2009) Running up Blueberry Hill: Prototyping Whole Body Interaction in Harmony Space In Proceedings of the Third Conference on Tangible and Embodied Interaction. ACM, New York, 2009, 92-98.

Holland, S., Bouwer,A, Dalgleish,M. and Hurtig, T. (2010) Feeling the Beat where it counts: Fostering Multi-limb Rhythm Skills with the Haptic Drum Kit. In Proceedings of TEI 2010 Boston Cambridge Mass.

Hurtienne, J. and Blessing, L. Design for Intuitive Use - Testing Image Schema Theory for User Interface Design. In Proceedings of the 16th International Conference on Engineering Design. (Paris, France, 2007). 1-12.

Hurtienne, J., Israel, J. H. and Weber, K. Cooking up Real World Business Applications Combining Physicality, Digitality, and Image Schemas. In Proceedings of the 2nd International Conference on Tangible and Embedded Interaction. (Bonn, 2008). ACM, New York, 2008, 239246

Johnson, M. Embodied Musical Meaning. Theory and Practice, 22-23 (1997-1998), 95-102.

Johnson, M. The philosophical significance of image schemas. In Hampe, B. and Grady, J. eds. From Perception to Meaning: Image Schemas in Cognitive Linguistics. Walter de Gruyter, Berlin, 2005, 15-33.

Klemmer, S. R., Hartmann, B., and Takayama, L. 2006. How bodies matter: five themes for interaction design. In Proceedings of the 6th Conference on Designing interactive Systems (University Park, PA, USA, June 26 - 28, 2006). DIS ‘06. ACM, New York, NY, 140-149.

Lakoff, G. and Johnson, M. Metaphors We Live By. The University of Chicago, London, 2003.

Lakoff, G. and Núñez, R. (2000) Where Mathematics Comes From: How the Embodied Mind Brings Mathematics into Being, Basic Books, New York.

Lakoff, G. (2002) Moral Politics: How Liberals and Conservatives Think. University of Chicago Press.

Larson, S. Musical forces and melodic patterns. Theory and Practice, 22-23 (1997-1998), 55-71.

O'Regan, K. and Noe, A. 2001, 'A Sensorimotor Account of Vision and Visual Consciousness', Behavioral and Brain Sciences 24(5), 883-917. 
Rohrer, T. The Body in Space: Dimensions of Embodiment. In Ziemke, T., Zlatev, J., Frank, R. and Dirven, R. eds. Body, Language, and Mind: Embodiment. Walter de Gruyter, Berlin, 2007, 339-378.

Saslaw, J. Forces, Containers, and Paths: The Role of Body-Derived Image Schemas in the Conceptualization of Music. Journal of Music Theory, 40, 2 (Autumn 1996), 217-243.

Van der Linden, J., Schoonderwaldt, E., Bird, J. and Johnson, R. (2010) MusicJacket - Combining Motion Capture and Vibrotactile Feedback to Teach Violin Bowing, IEEE Transactions on Instrumentation and Measurement.

Wilkie, K., Holland, S. and Mulholland, P. (2009) Evaluating Musical Software Using Conceptual Metaphors. In Proceedings of the 23rd British Computer Society Conference on Human Computer Interaction. (Cambridge, 2009). British Computer Society, 2009, 232-237.

Wilkie, K., Holland, S. and Mulholland, P. (2010) What Can the Language of Musicians Tell Us about Music Interaction Design? Computer Music Journal CMJ 34(4)

Zbikowski, L. M. Conceptual Models and Cross-Domain Mapping: New Perspective on Theories of Music and Hierarchy. Journal of Music Theory, 41, 2 (Autumn 1997), 193-225.

Zbikowski, L. M. Metaphor and music theory: Reflections from cognitive science. Music Theory Online, 4, (1998), 1-8. 